\title{
Aftermath Earthquake in Nepal: Burden of Scrub Typhus Cases and their Presentations
}

\author{
Bastola $\mathbf{A}^{1 *}$, Marahatta $\mathbf{S B}^{2,3}$, Jha $\mathbf{S}^{1}$ and Pant $\mathbf{N}^{1}$ \\ ${ }^{1}$ Sukraraj Tropical and Infectious Disease Hospital, Kathmandu, Nepal \\ ${ }^{2}$ Manmohan Memorial Institute of Health Science, Kathmandu, Nepal \\ ${ }^{3}$ Institute of Public Health, Liverpool John Moores University, UK
}

*Corresponding author: Dr. Anup Bastola, Sukraraj Tropical and Infectious Disease Hospital, Kathmandu, Nepal, Tel: +86 1058900739; E-mail: docanup11@gmail.com Received date: April 04, 2017; Accepted date: April 22, 2017; Published date: April 30, 2017

Copyright: () 2017 Anup Bastola, et al. This is an open-access article distributed under the terms of the Creative Commons Attribution License, which permits unrestricted use, distribution, and reproduction in any medium, provided the original author and source are credited.

\begin{abstract}
Background: Scrub typhus, also known as tsutsugamushi disease, is an infectious illness caused by Orientia (Rickettsia) tsutsugamushi. It is widely found in Asian counztries and human beings of all ages including children are influenced by it. It can cause death due to late presentation, delayed diagnosis and drug resistance. The clinical features and complications of scrub typhus vary from mild to fatal illness. We describe the epidemiology, clinical features and treatment outcome among scrub typhus cases admitted in Sukraraj Tropical and Infectious Disease Hospital (STIDH), a tertiary center in Kathmandu, Nepal following the massive earthquake of 2015.
\end{abstract}

Material and methods: Serum samples of twenty-three patients with acute undifferentiated fever admitted to the STIDH were tested positive for orientia tsutsugamushi by IgM ELISA. These patients were admitted between August to October 2015. Detail history taking, clinical evaluation and laboratory parameters of these patients were collected. Most of the patients were from the earthquake effected district and staying in temporary shelter with history of rodent infestation of the environment. Verbal consent was taken from each patient. The data were entered in SPSS version 16 and descriptive statistics was used to analyze the data.

Results: In the study, more than half of the patients $(52.2 \%)$ were female and the mean $( \pm S D)$ age of the patients was $37.6( \pm 13.3)$ years. Most of the patients $(82.7 \%)$ were engaged in farm work. About three fifths $(60.8 \%)$ of the patients were from Dhading district followed by Nuwakot, Sarlahi, Kavre and Parsa. Except Sarlahi district, all other were affected by earthquake 2015 . Majority $(87 \%)$ of the patients were living in the temporary shelter following devastating earthquake and had history of environmental infestation by rodents. All the patients had fever and anorexia. The mean fever duration before admission was $10.1( \pm 4.0)$ days. Clinical features of arthralgia and myalgia $(91.3 \%)$; nausea, headache and chills or rigors $(82.6 \%)$ and retroorbital pain $(60.9 \%)$ among the commonest. Abdominal pain and cough was complained by $47.8 \%$ and $43.5 \%$ patients respectively. Eschar formation, red eye and lymphadenopathy were the commonest physical finding and noted in $30.4,30.4 \%$ and $26.1 \%$ patients respectively. Lymphadenopathy was localized. Most common laboratory parameter was increased in alanine transaminase level and thrombocytopenia and was seen in $73.9 \%$ and $60.9 \%$ patients respectively. Leukocytosis was seen in $21.7 \%$ of patients. Azithromycin or doxycycline was added on ceftriaxone once the diagnosis of scrub typhus was made. The mean fever response time was $1.7( \pm 1.2)$ days. All patients were discharged after fever subsides. Clinical recovery and hospital stay was uneventful.

Conclusion: From the study, it can be concluded that scrub typhus has emerged as an important cause of febrile illness in Nepal after massive earthquake of 2015 so; it demands the continuous surveillance in the health care setting. Scrub typhus should be considered in the patient presenting with acute undifferentiated fever and can be confirmed by sensitive test as characteristic clinical findings seen only in small number of cases. Rodent infestation of the environment around temporary shelter increases the risk of acquiring scrub typhus and such history increases clinical suspicion in the diagnosis. Azithromycin and doxycycline are the effective antibiotics. Appropriate diagnosis and treatment can reduce the complication of scrub typhus even in resource-limited environment.

Keywords: Scrub typhus; Undifferentiated fever; Transaminitis; Thrombocytopenia

\section{Introduction}

Scrub typhus, also known as tsutsugamushi disease, is an infectious illness caused by Orientia (Rickettsia) tsutsugamushi, transmitted to humans and rodents by the bite of the larva of trombiculid mites (chigger). The mites are both the vector and reservoir of the disease.
The mite is very small and can only be seen through a microscope or magnifying glass. The larva is the only stage that can transmit the disease to humans and other vertebraes [1-3]. The disease is transmitted from mites to 'rats and mice' and the mites in their larval stage contract the disease organism by biting these rodents. Humans are the accidental host. The disease is not directly transmitted from person to person. Human beings of all ages including children are influenced by it [4]. 
Scrub typhus was first described from Japan in 1899 where it was found to be transmitted by mites. It is a Zoonotic disease and widespread disease in Asia and Pacific Islands. It also occurs in Japan, South Korea, Nepal, Northern Pakistan, South China, Papua New Guinea, and the Australian states of Queensland and Northern New South Wales [5]. Geographically when scrub typhus is confined to the Asia Pacific region, a billion of people are at danger and every year, almost a million of cases are reported [4]. The burden of disease in rural Asia is high, with studies presenting scrub typhus causing up to $20 \%$ of febrile hospital admissions [6].

Infected chiggers are specifically found during the wet season in the dense areas of scrub vegetation which is when the mite lay their eggs [7]. Areas like forest clearings, riverbanks, and grassy regions provide optimal conditions for the infected mites to thrive [1]. The southern terai region of Nepal is a suitable environment for scrub typhus [8].

According to WHO, 1999, scrub typhus is probably one of the most underdiagnosed and underreported febrile illness requiring hospitalization in the region. The absence of definitive sign and symptoms combined with a general dependence upon serological tests make the differentiation of scrub typhus from other common febrile diseases (murine typhus, typhoid fever and leptospirosis) quite difficult [9]. The clinical features and complications of scrub typhus vary from mild to fatal illness [10].

The early manifestations are an eschar, representing localized cutaneous necrosis at the site of mite feeding (which is not always present) and regional lymphadenopathy followed by fever, headache, myalgia, generalised lymphadenopathy,cough,gastrointestinal symptoms, rash, transient hearing loss and conjunctival injection [11].

The life threatening complications of this disease are jaundice, renal failure, pneumonitis, acute respiratory distress syndrome (ARDS), septic shock, myocarditis and meningo-encephalitis. The complications of scrub typhus usually develop after the 1st week of illness [12].

The diagnosis of this disease may be confirmed by a laboratory test such as serology. Among the various serological methods, Weil-Felix test is the cheapest and most easily available but notoriously unreliable. Indirect immunofluorescence antibody (IFA) is the gold standard. As compared to rodents. Humans are the accidental host. The disease is not directly transmitted from person to person. Human beings of al ages including children are influenced by it [4]

Scrub typhus was first described from Japan in 1899 where it was found to be transmitted by mites [1]. It is a Zoonotic disease and widespread disease in Asia and Pacific Islands. It also occurs in Japan, South Korea, Nepal, Northern Pakistan, South China, Papua New Guinea, and the Australian states of Queensland and Northern New South Wales [5].

Geographically when scrub typhus is confined to the Asia Pacific region, a billion of people are at danger and every year, almost a million of cases are reported [4]. The burden of disease in rural Asia is high, with studies presenting scrub typhus causing up to $20 \%$ of febrile hospital admissions [6].

Infected chiggers are specifically found during the wet season in the dense areas of scrub vegetation which is when the mite lay their eggs [7]. Areas like forest clearings, riverbanks, and grassy regions provide optimal conditions for the infected mites to thrive [1]. The southern terai region of Nepal is a suitable environment for scrub typhus [8]
The diagnosis of this disease may be confirmed by a laboratory test such as serology. Among the various serological methods, Weil-Felix test is the cheapest and most easily available but notoriously unreliable. Indirect immunofluorescence antibody (IFA) is the gold standard. As compared to commercial test kits, ELISA provides more sensitivity and equal specificity. The available antibiotics like doxycycline and azithromycin has good efficacy to scrub typhus [1].

After the 7.8 and 7.3 magnitude earthquake struck in Nepal on 25 April and 12 May, 2015 respectively and the subsequent strong aftershocks, outbreaks of scrub typhus were reported from various parts of the country, especially from districts affected by the earthquake $[13,14]$.

These outbreaks were thought to be due to people and rodents (which carry mites with the bacterium Orientiatsutsugamushi, the infective microbe in scrub typhus) living in close proximity in temporary shelters after the earthquake.

As revealed by the post-earthquake outbreaks in Nepal, scrub typhus can be deadly because it caused dozens of deaths [15]. It can cause death due to late presentation, delayed diagnosis and drug resistance $[4,16,17]$. It is necessary to be aware of its early signs and symptoms. This study was conducted to describe the epidemiology and clinical features of scrub typhus cases seen in a tertiary infectious diseases hospital of Nepal following the massive earthquake of 2015.

\section{Materials and Methods}

We describe the demographic data, clinical profile, laboratory findings and treatment outcome of 23 scrub typhus cases who were admitted in Sukraraj Tropical and Infectious Disease Hospital, Teku Kathmandu Nepal between August to October 2015.

They were the individuals rendered homeless in the most devastated districts and living in temporary shelters with a high reported incidence of mouse infestation. The ethical permission was taken from Sukraraj Tropical and Infectious Disease Hospital to execute the study.

All patients were evaluated clinically. Further, routine laboratory tests like complete blood count, renal function tests, liver function test, blood culture and serology test for other febrile illnesses were done. Treatment outcome with antibiotics also recorded.

Moreover, diagnosis of scrub typhus was done by using IgM Elisa (In Bios, USA) at national public health laboratory (NPHL). Serum sample from one patients whose IgM Elisa was positive from our center was sent for the confirmation to AFRIM Bangkok along with other twelve samples from other center. The serum sample from this patients was positive for scrub typhus by RT PCR (Ct- 34.54), dot Elisa /IgG/IgM, IFA) IgM (1:3200), IgG (1:400).

The data were entered in SPSS version 16 and descriptive statistics was used to analyze the data. Verbal consent was obtained from each patient. The identity and information of the patients were kept confidential and not disclosed to anyone.

\section{Results}

A total of 23 cases with undifferentiated fever were diagnosed of Scrub typhus by IgM Elisa. Out of them more than half of the respondents $52.2 \%$ were male while $47.8 \%$ were female. 
Page 3 of 5

The Mean $( \pm \mathrm{SD})$ age of the patients was $37.6( \pm 13.3)$ years and ranges from 16 to 65 years. Most of the patients $82.7 \%$ were farmer followed by student (13\%) and teacher $(4.3 \%)$ by profession (Figure 1 ).

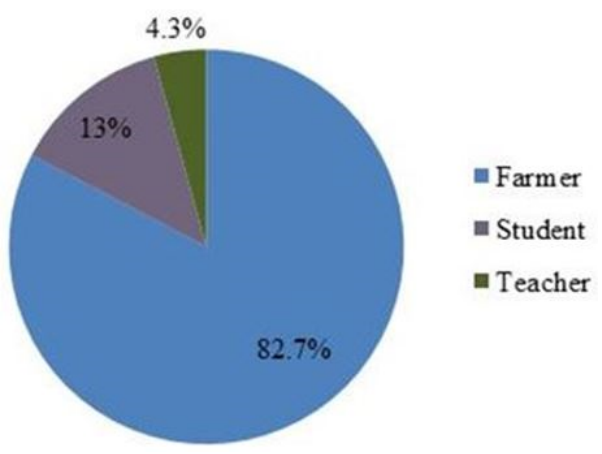

Figure 1: Professions.

Similarly, about three fifths of the patients $60.8 \%$ were from Dhading district followed by Nuwakot (21.7\%), Sarlahi $(8.6 \%)$ and (4.3\%) each from Kavre and Parsa.

Majority $87 \%$ of the patients were living in the temporary shelter following devastating earthquake and one in ten patients (13\%) were living in the normal house. All of them had different livestock including cow, buffalo, goat or in combination nearby home.

Those who were staying in temporary shelter had livestock kept in a few meter distances. All of the patients had history of the contact with mice. There was history of increased mice population in their household.

Fever was the most common finding among all the patients and it was associated with chills and rigor among $82.6 \%$ of the patients. The mean $( \pm \mathrm{SD})$ fever duration was of $10.1( \pm 4.0)$ days and ranges from 4 to 21 days.

All of the patients complained of anorexia (loss of appetite). Arthralgia and myalgia was present in $91.3 \%$ of the patients. Headache was present in about four fifth of the patients (82.6\%) and associated with retro orbital pain among three fifth of the patients (60.9\%).

Cough was present in four-in-ten patients (43.5\%) and was associated with sputum production among nearly a quarter of the patients $(21.7 \%)$.

Similarly, abdominal pain, nausea, vomiting was present in $47.8 \%$, $82.6 \%$ and $26.1 \%$ of the patients respectively. Moreover, only $8.7 \%$ of the patients complained of burning micturition.

On clinical evaluation, more than one fourth of the patients i.e., $30.4 \%$ had red eye. Similarly, $30.4 \%$ of the patients had typical eschar which was present in $28.5 \%$ patients on the posterior part and right side of the neck; in $28.5 \%$ patient's left and right anterior axillary line, in $14.2 \%$ patient in right chest; in $14.2 \%$ patient in right inguinal region and in $14.2 \%$ patient in right leg.

Likewise, localized lymphadenopathy was seen among $26.1 \%$ of patients. Lung crepitation and splenomegaly was present in only $8.6 \%$ patients. Just $4.3 \%$ of the patients had rheumatic heart disease and had cardiac murmur (Table 1).

\begin{tabular}{|c|c|c|}
\hline \multicolumn{3}{|l|}{ Symptoms of scrub typhus } \\
\hline Symptoms & Frequency & Percent \\
\hline Fever & 23 & 100 \\
\hline Chills/Rigor & 19 & 82.6 \\
\hline Arthralgia & 21 & 91.3 \\
\hline Myalgia & 21 & 91.3 \\
\hline Headache & 19 & 82.6 \\
\hline Retro-orbital pain & 14 & 60.9 \\
\hline Cough & 10 & 43.5 \\
\hline Sputum production & 5 & 21.7 \\
\hline Abdominal pain & 11 & 47.8 \\
\hline Nausea & 19 & 82.6 \\
\hline Vomiting & 6 & 26.1 \\
\hline Anorexia (Loss of appetite) & 23 & 100 \\
\hline Burning Micturition & 2 & 8.7 \\
\hline \multicolumn{3}{|l|}{ Signs of scrub typhus } \\
\hline Red eye & 7 & 30.4 \\
\hline Lymphadenopathy & 6 & 26.1 \\
\hline Crept/Ronchi & 2 & 8.7 \\
\hline Cardiac Murmur & 1 & 4.3 \\
\hline Splenomegaly & 2 & 8.6 \\
\hline Eschar & 7 & 30.4 \\
\hline
\end{tabular}

Table 1: Clinical sign and symptoms of scrub typhus.

Laboratory examination revealed the mean $( \pm \mathrm{SD})$ hemoglobin level $11.8( \pm 1.0) \mathrm{gm} \%$ and ranges from 10.2 to $13.9 \mathrm{mg} \%$. Lymphocyte count was higher than 10000 cells $/ \mathrm{cmm}$ present in about one fifth of the patients $(21.7 \%)$ and ranging from 4100 to 16700 cells/cmm. Alanine aminotransferase (ALT) was increased from the upper limit (>40 IU/L) among nearly three fourth of the patients $(73.9 \%)$ with mean $( \pm \mathrm{SD})$ of $69.8( \pm 50.5)$ and ranges from 21 to $211 \mathrm{IU} / \mathrm{L}$. Thrombocytopenia (platelets counts $<150000$ cells $/ \mathrm{cmm}$ ) was present among about three fifth of the patients (60.9\%) with range from 70000 to 25200 cells/ $\mathrm{cmm}$. Further, nearly two fifth of the patients i.e., $39.1 \%$ had mild (149999-100000) and nearly a quarter of the patients (21.7\%) had moderate $(<100000-50000)$ thrombocytopenia. Blood culture was no growth in all the patients.

All of the patients were treated with antibiotics. Majority $91.3 \%$ of the patients were treated with Ceftriaxone and only $8.6 \%$ were treated with Ceftriaxone and Sulbactum. Nearly one fifth of the patients i.e., $17.3 \%$ responded to only Ceftriaxone. Other patients added with doxycycline or azithromycin.

About three fifth of the patients (60.8\%) added with doxycycline $100 \mathrm{mg}$ twice a day. Furthermore, about one fifth of the patients i.e., $21.7 \%$ added with azithromycin once a day. The mean fever response time after use of Azithromycin or Doxycycline or Ceftriaxone was 1.7 
$( \pm 1.2)$ days. $17.4 \%$ didn't develop fever in hospital. More than one fourth of the patients (34.8\%) responded fever in one day followed by $26.1 \%$ patients in 2 days, $13 \%$ patients in 3 days and $8.7 \%$ patients in 4 days.

\section{Discussion}

Scrub typhus is responsible for a large proportion of undifferentiated fevers in south-east Asia [18]. Nepal has diversity in the landscape extending from north to south from high Himalayas, mountain area followed by the flatland. This typical landscape gives the opportunities for the vector to survive in rodents. Scrub typhus was reported from the studies done among patients with febrile illness in the Kathmandu valley. However the large numbers of cases were presented and diagnosed following massive Gorkha Earthquake 2015.

The availability of the diagnostic test this year in National Public Health Laboratories contributed for the diagnosis. Before this there was no test available for diagnosis of scrub typhus in government system. Diagnosis of all the cases was based on scrub typhus IgM elisa (InBios USA). However, during the outbreak for the confirmation of scrub typhus thirteen samples were send to AFRIM Bangkok from the epidemiology and disease control division (EDCD) Nepal.

Thirteen samples from all around the country was send and five came out to be positive by polymerase chain reaction, dot Elisa and indirect fluorescent antibody tests. One of the sample was from our case was included and was positive for scrub typhus by RT PCR (Ct34.54), dot Elisa / IgG/IgM, IFA) IgM (1:3200), IgG (1:400). We rule out the other causes of undifferentiated fever and most of the patients were negative for dengue, chikungunya, rapid diagnostic test for Malaria, Leptospira. Blood culture was also negative for almost all patients.

In the study, about three fifths of the patients $60.8 \%$ were from Dhading district followed by Nuwakot, Sarlahi, Kavre and Parsa. Except Sarlahi, all other district was the most affected district by earthquake 2015. 13 out of 23 cases diagnosed in the hospital, majority $87 \%$ of the patients were residing in their temporary shelter and gives history of increased rodents' number within and in vicinity of the resident.

Most of the patients $82.7 \%$ were engaged in the farm work. This is similar to the result of the study conducted [19]. By their study, the diagnosed scrub typhus patients were predominantly farmers.

In our study, the most common clinical symptom of scrub typhus was fever. Cent percent of the patients presented with undifferentiated fever which is consistent with the result of the study conducted $[20,21]$ and they had found fever in $100 \%$ of the patients.

Similarly, Ogawa [22] had found fever in $98 \%$ of the patients. In the study, most of the undifferentiated fever had chills and rigor. Before presenting to the hospital the mean duration of fever was 10 days. All of the patients presented with loss of appetite could be because of high grade fever. Arthralgia and myalgia was among other common complaints. More than ninety percent of the patients complained of these symptoms. Headache and Retro orbital pain were also other complaints by the patients. Four-in-ten patients (43.5\%) presented with cough and nearly a quarter of the patients $(21.7 \%)$ had sputum production. None of these patients developed any complications related to chest.
Among the gastrointestinal symptoms nausea was the most common finding. About two fifths of the patients (47.8\%) complained abdominal pain and $26.1 \%$ of the patients had vomiting. There were no complications related to gastrointestinal symptoms. Only $8.7 \%$ of the patients presented with burning micturition.

On clinical evaluation, all of 23 patients had stable vitals on admission. More than one fourth of the patients (30.4\%) had characteristic conjunctival injection. This findings is similar to the findings of Kamarasu [23] carried out in Tamilnadu. Eschar was seen among $30.4 \%$ of the patients. This finding is less than the findings of the study done in Pondicherry where eschar was seen in $46 \%$ cases [24].

Lymphadenopathy was seen among about a quarter of patients (26.1\%) and was associated to the site of eschar however generalized lymphadenopathy was not seen in all cases. This finding is contrast to the other findings as $30 \%$ of patient of patient had generalized lymphadenopathy as per findings of the study done in Pondicherry [24]. Splenomegaly was revealed in only $8.7 \%$ patients after ultrasonography of the abdomen however clinically this was not present.

Similarly, $8.7 \%$ of the patients presented with crepitation and rhonchi. Just $4.3 \%$ of the patient had chronic obstructive pulmonary disease. Likewise, $4.3 \%$ of the patient who was 16 years old had rheumatic heart disease and presented with murmur. No cardiac complication developed during the stay in hospital.

Lymphocytosis was seen in about one fifth of the patients (21.7\%). Lymphocytosis is less common in our patients; it was described in higher number of patients in South Vietnam [25]. Alanine aminotransferase was higher than the upper baseline $(>40 \mathrm{IU} / \mathrm{L})$ among nearly three fourth of the patients $(73.9 \%)$ however increase in serum bilirubin was not seen in any of the patients. Thrombocytopenia was seen among about three fifth of the patients (60.9\%). Further, nearly a quarter of the patients (21.7\%) had moderate thrombocytopenia. None of the patients developed bleeding.

Patients responded to Azithromycin $(500 \mathrm{mg}$ ) daily for five days or doxycycline $100 \mathrm{mg}$ twice a day for 10 days. Four patients responded to ceftriaxone; however, their past antibiotic intake could not be assessed. Azithromycin and Doxycycline are sensitive in Nepalese Scrub typhus. None of the patients developed any complication associated with the disease. This could be because of immediate referral of serious cases to center where ICU available before their reports available and lack of follow up. We had referral of many serious cases of undifferentiated fever during that period.

\section{Conclusion}

From the study, it can be concluded that scrub typhus has emerged as an important cause of febrile illness in Nepal after massive earthquake of 2015 so, it is necessary to continue inspection of it. The most common symptoms of scrub typhus were fever and anorexia. It should be considered in the patient presenting with fever, anorexia, chills or rigors, myalgia, arthralgia, headache, nausea and also if the patient living in temporary shelter and come in contact with mice. Azithromycin and doxycycline are the effective antibiotics for it. Appropriate diagnosis and treatment can reduce the complication of scrub typhus even in resource-limited environments. 
Citation: Bastola A, Marahatta SB, Jha S, Pant N (2017) Aftermath Earthquake in Nepal: Burden of Scrub Typhus Cases and their Presentations. J Trop Dis 5: 236. doi:10.4172/2329-891X.1000236

Page 5 of 5

\section{Acknowledgement}

We would like to express our gratitude to Sukraraj Tropical and Infectious Disease Hospital for providing opportunity to conduct the study. Special thanks to Dr Leo Liu and Amrita Chaulagain for their immense help.

\section{References}

1. B Basnyat (2016) Typhoid versus typhus fever in post-earthquake Nepal Lancet 4(8): 516-517.

2. Rapsang AG, Bhattacharyya P (2013) Scrub typhus. Indian J Anaesth 57: 127-134.

3. Ministry of Health and Population (2016) Infectious disease control guideline. Government of Nepal.

4. Watt G (2003) Scrub typhus. In: Warrell DA, Cox TM, Firth JD, Benz EJ (eds.). Oxford textbook of medicine (4th edn.). Oxford University Press, Oxford 6: 629-631.

5. Mathai E, Lloyd G, Cherian T, Abraham OC, Cherian AM, et al. (2001) Serological evidence for the continued presence of human rickettsioses in southern India. Ann Trop Med Parasitol 95: 395-398.

6. Brown GW, Robinson DM, Huxsoll DL, Ng TS, Lim KJ, et al. (1976) Scrub typhus: A common cause of illness in indigenous populations. Trans R Sco Trop Med Hyg 70: 444-448.

7. Bechah Y, Capo C, Mege JL, Raoult D (2008) Epidemic typhus. Lancet Infect Dis 8: 417-426.

8. Brown GW, Shirai A, Gan E, Bernthal P (1981) Antibodies to typhus in Eastern Nepal. Trans Roy Soc Trop Med Hyg 75: 586-587.

9. World Health Organization (1999) WHO Recommended Surveillance Standards (2nd edn.). WHO, Geneva. pp: 123-124.

10. Jeong YJ, Kim S, Wook YD, Lee JW, Kim KI, et al. (2007) Scrub typhus: Clinical, pathologic and imaging findings. Radiographics 27: 161-172.

11. Lee N, Ip M, Wong B, Lui G, Tsang OTY, et al. (2008) Risk factors associated with life threatening rickettsial infections. Am J Trop Med Hyg 78: 973-978.

12. Subbanna PKA, Suri SD (2012) Multi-organ dysfunction in scrub typhus. Ann Trop Med Public Health 5: 393-396.
13. http://www.unicef.org/about/annualreport/files/Nepal_2015_COAR.pdf

14. http://kathmandupostekantipur.com/news/2015-10-07/rats-causingscrub-typhus-who-team.html

15. Basnyat B (2016) Typhoid versus typhus fever in post-earthquake Nepal. The Lancet Global Health 4(8): 516-517.

16. Cowan GO (2003) Rickettsial infections in manson's tropical diseases. Elsevier Science, London. pp: 891-906.

17. Tamura A, Ohashi N, Urakami H, Miyamura S (1995) Classification of Rickettsia tsutsugamushi in a new genus, Orientia gen. nov., as Orientiatsutsugamushi comb.nov. Int J Syst Bacteriol 45(3): 589-591.

18. Paris DH, Jenjaroen K, Blacksell SD, Phetsouvanh R, Wuthiekanun V, et al. (2008) Differential patterns of endothelial and leucocyte activation in typhus-like illness in Laos and Thailand. Clin Exp Immunol 153(1): 63-67.

19. Hu J, Tan Z, Ren D, Zhang X, He Y, et al. (2013) Clinical characteristics and risk factors of an outbreak with scrub typhus in previously unrecognized areas, Jiangsu province, China. PLOS One 10(5): e0125999.

20. Sharma R, Krishna VP, Manjunath, Singh H, Shrivastava S, et al. (2014) Analysis of two outbreaks of scrub typhus in Rajasthan: A clinicoepidemiological study. J Assoc Physicians India 62(12): 24-29.

21. Jim NT, Chiu NC, Chan WT, Ho CS, Chang JH, et al. (2009) Clinical manifestations, laboratory findings and complications of pediatric scrub typhus in eastern Taiwan. Pediatr Neonatal 50(3): 96-101.

22. Ogawa M, Hagiwara T, Kishimoto T, Shiga S, Yoshida Y, et al. (1998) Scrub typhus in Japan: Epidemiology and clinical features of cases reported. Am J Trop Med Hyg 67(2): 162-165.

23. Kamarasu K, Mathan M, Rajagopal V, Subramaniam K (2007) Serological evidence for wide distributioin of spotted fevers and scrub typhus fever in Tamil Nadu. Indian J Med Res 126: 128-130.

24. Vivekanandan M, Mani A, Priya Y, Singh A, Jayakumar S, et al. (2010) Outbreak of scrub typhus in Pondicherry. J Assoc Physicians India 58: 24-28

25. Berman SJ, Kundin WD (1973) Scrub Typhus in South Vietnam. A study of 87 cases. Ann Intern Med 79: 26-30. 